\title{
Warm-Logamediate inflationary universe model
}

\author{
Ramón Herrera* and Marco Olivarest \\ Instituto de Física, Pontificia Universidad Católica \\ de Valparaíso, Casilla 4059, Valparaíso, Chile.
}

(Dated: August 20, 2018)

\begin{abstract}
Warm inflationary universe models in the context of logamediate expansion are studied. General conditions required for these models to be realizable and discussed. This study is done in the weak and strong dissipative regimes. The parameters of our models are constrained from the observational data.
\end{abstract}

PACS numbers: $98.80 . \mathrm{Cq}$

${ }^{*}$ Electronic address: ramon.herrera@ucv.cl

${ }^{\dagger}$ Electronic address: marco.olivares@ucv.cl 


\section{INTRODUCTION}

In modern cosmology our notions concerning the early universe have introduced a new element, the inflationary scenario of the universe[1], which provides an attractive epoch for solving some of the problems of the standard big bang model, like the flatness, horizon etc..It is well known Inflation can provide an elegant mechanism to explain the large-scale structure [2] and a causal interpretation of the origin of the observed anisotropy of the cosmic microwave background (CMB) radiation[3, 4] .

On the other hand, it is well know that warm inflation, as opposed to the conventional cool inflation, has the attractive feature that it avoids the reheating period at the end of the accelerated expansion because of the decay of the inflaton field into radiation and particles during the slow roll phase [5]. During the warm scenario the dissipative effects are important, so that radiation production occurs concurrently together with the inflationary expansion. The dissipating effect arises from a friction term which describes the processes of the scalar field dissipating into a thermal bath. This scenario has additional advantages, namely: i) the thermal fluctuations during inflation may play a dominant role in producing the initial fluctuations necessary for Large-Scale Structure (LSS) formation. These density fluctuations arise from thermal rather than quantum fluctuations [6, 7], ii) the slow-roll conditions can be satisfied for steeper scalar potentials, iii) the universe stops inflating and "smoothly" enters in a radiation dominated Big-Bang phase [5, 8], iv) it may contribute a very interesting mechanism for baryogenesis, where the spontaneous baryo/leptogenesis can easily be realized in this scenario [9]. Recently, a new mechanism with a baryon asymmetry from dissipative effects during warm baryogenesis was studied in Ref.[10].

In this paper, we will examine warm inflation and the effective potentials that arise to study logamediate inflationary universe model where the dissipative effect is present. The logamediate model was originally examined by Barrow in Ref.[11]. During logamediate inflation, the scale factor $a(t)=\exp \left(A[\ln t]^{\lambda}\right)$, with $A>0$ and $\lambda>1$ constants. In particular,

for $\lambda=1$, this scale factor reduces to power law inflation i.e. $a \sim t^{A}[12]$. This accelerate expansion of the universe has the interesting property that the ratio of tensor to scalar perturbations is small and the power spectrum can be either red or blue tilted, according to the values of the parameters appearing in the model [13].

On the other hand, the condition for inflation to occur is that the inflaton field slow-roll 
near the top of the potential for sufficiently long time, so that the vacuum energy drives the inflationary expansion of the Universe. Many models of inflation have been proposed , based on single field or multi-field potentials see e.g. Refs.[14, 15]. We found that for the case of the weak dissipative regime, the field potential is $V(\phi) \propto \phi^{\alpha} \exp \left[-\beta \phi^{\gamma}\right]$ and this kind of potential coincides with the effective potential found by Barrow in Ref.[11] . In particular, when $\alpha=0$ this $V(\phi)$ includes exponential potential that appears in Kaluza-Klein theories, supergravity, and in super-string models (see Ref.[16]). When $\beta=0$ it potential includes power-law potentials[17]. In Ref.[18] was considered the behavior of the parameters $\gamma$ and $\alpha$ in all regions of the parameter space. We also find this sort of potentials in higherdimensional theories, scalar-tensor theories, and supergravity corrections [19]. Also, in the context of the curvaton reheating for this potential was studied in Ref.[20] and also the form of this potential was used in dark energy models[21]. Also, we found that for the case of the strong dissipative regime, the scalar field potential $V(\phi)$ is a function of the inverse gamma

function $\gamma_{\lambda}^{-1}\left[\frac{1}{\alpha_{1}} \ln \phi\right]$. This sort of potentials was analyzed in Refs. [22], for two models of nonlocal scalar fields on cosmological backgrounds (see also, Ref.[23]).

Thus, our aim in this paper is to analyze an evolving logamediate scale factor in the warm inflationary universe scenario. We will do this for two regimes; the weak and the strong dissipative regimes.

The outline of the paper goes as follow: The next section presents a short description of model. In the sections III and VI, we discuss the weak and strong dissipative regimes in the logamediate scenario. In both sections, we give explicit expressions for the dissipative coefficient, the scalar potential, the scalar power spectrum and the tensor-scalar ratio for these models. At the end, section $\mathrm{V}$ exhibits our conclusions. We chose units so that $c=\hbar=1$.

\section{THE WARM-INTERMEDIATE INFLATIONARY PHASE.}

We start by considering the flat Friedmann equation, by using the Friedmann-Robertson Walker (FRW) metric, where

$$
H^{2}=\frac{\kappa}{3} \rho=\frac{\kappa}{3}\left[\rho_{\phi}+\rho_{\gamma}\right]
$$


here, $\rho=\rho_{\phi}+\rho_{\gamma}$ represents the total energy density and the universe is filled with a self-interacting scalar field of energy density $\rho_{\phi}$ and a radiation field with energy density $\rho_{\gamma}$. The parameter $H=\dot{a} / a$ denotes the Hubble parameter, $a$ is the scale factor and $\kappa=8 \pi G=8 \pi / m_{p}^{2}$, where $m_{p}$ represents the Planck mass. In the following, also we will assume that the energy density associated to the scalar field is $\rho_{\phi}=\dot{\phi}^{2} / 2+V(\phi)$ and $V(\phi)=V$ is the scalar potential.

The dynamics equations for $\rho_{\phi}$ and $\rho_{\gamma}$ in the warm inflationary scenario is described by [5]

$$
\dot{\rho_{\phi}}+3 H\left(\rho_{\phi}+P_{\phi}\right)=-\Gamma \dot{\phi^{2}},
$$

and

$$
\dot{\rho}_{\gamma}+4 H \rho_{\gamma}=\Gamma \dot{\phi}^{2}
$$

Here $\Gamma>0$ is the dissipation coefficient and it is responsible of the decay of the scalar field into radiation during the inflationary era. This coefficient can be assumed to be a constant or a function of the scalar field $\phi$, or the temperature $T$, or both [5]. Dots mean derivatives with respect to time.

Following, Refs. [5, 6] in the inflationary scenario the energy density associated to the scalar field $\rho_{\phi}$ dominates over the energy density associated to the radiation field $\rho_{\gamma}$ and the Eq.(1) becomes

$$
H^{2} \approx \frac{\kappa}{3} \rho_{\phi}
$$

We also consider that during this scenario the radiation production is quasi-stable [5, 6], i.e. $\dot{\rho}_{\gamma} \ll 4 H \rho_{\gamma}$ and $\dot{\rho}_{\gamma} \ll \Gamma \dot{\phi}^{2}$. From Eq.(3) we obtained that the energy density of the radiation field is given by

$$
\rho_{\gamma}=\frac{\Gamma \dot{\phi}^{2}}{4 H}=-\frac{\Gamma \dot{H}}{2 \kappa H(1+R)},
$$

where $R$ is the rate defined as

$$
R=\frac{\Gamma}{3 H}
$$

for the case of the weak (strong) dissipation regime, we have $R<1(R>1)$. Here, we noted that the energy density of the radiation field $\rho_{\gamma}$, could be written as $\rho_{\gamma}=C_{\gamma} T^{4}$, where $C_{\gamma}=\pi^{2} g_{*} / 30, g_{*}$ is the number of relativistic degrees of freedom and $T$ is the temperature of the thermal bath. 
Considering Eqs. (2) and (4), we can write

$$
\dot{\phi}^{2}=-\frac{2 \dot{H}}{\kappa(1+R)},
$$

and from Eqs.(5) and (7), the temperature of the thermal bath can be rewritten as

$$
T=\left[-\frac{\Gamma \dot{H}}{2 \kappa C_{\gamma} H(1+R)}\right]^{1 / 4} .
$$

On the other hand, the dissipation coefficient is computed for models in cases of lowtemperature regimes in Refs. [24, 25]. This dissipation coefficient, was developed in supersymmetric models which have an inflaton together with multiplets of heavy and light fields. Here, three super-fields $\Phi, X$ and $Y$ with a super-potential, $W=g \Phi X^{2}+h X Y^{2}$, where $\phi, \chi$ and $y$ refer to their bosonic component were study. The inflaton field couples to heavy bosonic field $\chi$ and fermions $\psi_{\chi}$, obtain their masses through couplings to $\phi$, where $m_{\psi_{\chi}}=m_{\chi}=g \phi$. Following Ref.[24], the transfer of energy from the inflaton the mass $m_{\phi}$ to another fields is dependent the coupling strength of the interaction, the relative sizes of the mass $m_{\phi}$, the mass of decay products and the temperature. The scenario of concern occurs when $m_{\chi}, m_{\psi_{\chi}}>T>H$ and $m_{y}, m_{\psi_{y}}<T$. These conditions specifies to here as the low-temperature scenario [24]. Considering this scenario, the coefficient $\Gamma$, when $X$ and $Y$ are singlets is given by

$$
\Gamma \simeq 0.64 g^{2} h^{4}\left(\frac{g \phi}{m_{\chi}}\right)^{4} \frac{T^{3}}{m_{\chi}^{2}}=C_{\phi} \frac{T^{3}}{\phi^{2}}
$$

where $C_{\phi}=0.64 h^{4} \mathcal{N}$ in which $\mathcal{N}=\mathcal{N}_{\chi} \mathcal{N}_{\text {decay }}^{2}$. Here, $\mathcal{N}_{\chi}$ is the multiplicity of the $X$ superfield and $\mathcal{N}_{\text {decay }}$ is the number of decay channels available in $X$ 's decay [24, 26] (see also, Refs. [27, 28]).

From Eqs.(8) and (9) we get

$$
\Gamma^{1 / 4}(1+R)^{3 / 4} \simeq \frac{\alpha_{0}}{\phi^{2}}\left[-\frac{\dot{H}}{H}\right]^{3 / 4},
$$

and so

$$
\Gamma \simeq \frac{\alpha_{0}^{4}}{\phi^{8}}\left[-\frac{\dot{H}}{H(1+R)}\right]^{3}
$$

where $\alpha_{0} \equiv C_{\phi}\left[\frac{1}{2 \kappa C_{\gamma}}\right]^{3 / 4}$. The Eq.(11) determines the dissipation coefficient in the weak (or strong) dissipative regime in terms of scalar field and the parameters of the model. 
Considering Eqs.(11) and (5) the scalar potential becomes

$$
V(\phi)=\frac{1}{\kappa}\left[3 H^{2}+\frac{\dot{H}}{(1+R)}\left(1+\frac{3}{2} R\right)\right],
$$

which could be expressed explicitly in terms of the scalar field, in the weak (or strong) dissipative regime.

Following Ref. [13], a generalized model of expansion of the universe is logamediate inflation. Here, the scale factor $a(t)$, is given by

$$
a=\exp \left[A(\ln t)^{\lambda}\right]
$$

where $\lambda$ and $A$ are dimensionless constants parameters such that $\lambda>1$, and $A>0$, see Ref.[13]. Recalling, that for $\lambda=1$ and $A=p$, the logamediate inflation model becomes power-law inflation, in which $a \sim t^{p}$.

In the following, we study models for a variable dissipation coefficient $\Gamma$, and we will restrict ourselves to the weak (or strong) dissipation regime.

\section{THE WEAK DISSIPATIVE REGIME.}

Assuming that, once the system evolves according to the weak dissipative regime, i.e. $\Gamma<3 H$ and considering Eqs.(7) and (13), we obtained a relation between the scalar field and cosmological times given by

$$
\phi(t)=\phi_{0}+\sqrt{\frac{2 A \lambda}{\kappa}}\left[\frac{2}{1+\lambda}\right](\ln t)^{\frac{1+\lambda}{2}},
$$

where $\phi_{0}$ is constant. The Hubble parameter as a function of the inflaton field, becomes

$$
H(\phi)=(A \lambda) B^{\lambda-1} \phi^{\gamma(\lambda-1)} \exp \left[-B \phi^{\gamma}\right],
$$

where

$$
\gamma \equiv \frac{2}{\lambda+1} \quad B \equiv\left[\frac{1}{\gamma} \sqrt{\frac{\kappa}{2 A \lambda}}\right]^{\gamma} .
$$

Without loss of generality the constant $\phi_{0}=0$.

From Eq.(12) the scalar potential in this regime is $V=\left(3 H^{2}+\dot{H}\right) / \kappa$. At late times and following Ref.[13], the scalar potential becomes

$$
V(\phi)=V_{0} \phi^{\alpha} \exp \left[-\beta \phi^{\gamma}\right],
$$


where $V_{0}=\frac{3}{\kappa}(A \lambda)^{2} B^{2(\lambda-1)}, \quad \alpha=2 \gamma(\lambda-1)$ and $\beta=2 B$.

Note that this kind of potential coincides with the scalar potential found in Ref.[13]. Note also that the scalar field $\phi$, the Hubble parameter $H$, and the potential $V(\phi)$ become independent of the parameters $C_{\phi}$ and $C_{\gamma}$ in this regime.

From Eq.(11) the dissipation coefficient $\Gamma$ as function of scalar field $\phi$, results

$$
\Gamma(\phi)=\alpha_{0}^{4} \phi^{-8} \exp \left[-3 B \phi^{\gamma}\right]
$$

Considering, the dimensionless slow-roll parameter $\varepsilon$, we get

$$
\varepsilon=-\frac{\dot{H}}{H^{2}}=(A \lambda)^{-1} B^{-(\lambda-1)} \phi^{-\gamma(\lambda-1)}
$$

and the other slow-roll parameter $\eta$, becomes

$$
\eta=-\frac{\ddot{H}}{H \dot{H}}=\left(A \lambda B^{\lambda}\right)^{-1} \phi^{-\gamma \lambda}\left[2 B \phi^{\lambda}-(\lambda-1)\right] .
$$

So, the condition for inflation to occur $\ddot{a}>0$ (or equivalently $\varepsilon<1$ ) is only satisfied when $\phi>\left[A \lambda B^{(\lambda-1)}\right]^{\frac{-1}{\gamma(\lambda-1)}}$.

The number of e-folds $N$ between two different values of cosmological times $t_{1}$ and $t_{2}$ or equivalently between two values of the scalar field $\phi_{1}$ and $\phi_{2}$, from Eq.(14) results

$$
N=\int_{t_{1}}^{t_{2}} H d t=A\left[\left(\ln t_{2}\right)^{\lambda}-\left(\ln t_{1}\right)^{\lambda}\right]=A B^{\lambda}\left(\phi_{2}^{\gamma \lambda}-\phi_{1}^{\gamma \lambda}\right) .
$$

Following Ref.[13] and considering that inflation begins at the earliest possible stage, where $\varepsilon=1$ or equivalently $\ddot{a}=0$, the scalar field $\phi_{1}$, is given by

$$
\phi_{1}=\left[A \lambda B^{(\lambda-1)}\right]^{\frac{-1}{\gamma(\lambda-1)}}
$$

On the other hand, the density perturbation could be written as $\mathcal{P}_{\mathcal{R}}{ }^{1 / 2}=\frac{H}{\dot{\phi}} \delta \phi[5]$. In particular in the warm inflation regime, a thermalized radiation component is present, therefore, inflation fluctuations are dominantly thermal rather than quantum [5, 6]. In the weak dissipation limit, we have $\delta \phi^{2} \simeq H T$ [6, 29]. From Eqs.(17) and (으), the density perturbation is given by

$$
\mathcal{P}_{\mathcal{R}}=\beta_{4} \phi^{\alpha-2} \exp \left[-\beta \phi^{\gamma}\right]
$$

where

$$
\beta_{4}=\frac{1}{4}\left(\frac{C_{\phi}}{C_{\gamma}}\right)(A \lambda)^{2} B^{2(\lambda-1)}
$$


The scalar spectral index $n_{s}$ is given by $n_{s}-1=\frac{d \ln \mathcal{P}_{\mathcal{R}}}{d \ln k}$. Here, the interval in wave number $k$ is related to the number of e-folds $N$, through $d \ln k(\phi)=d N(\phi)=(H / \dot{\phi}) d \phi$. From Eqs. (14) and (22), we get

$$
n_{s}=1-\frac{2 B^{-(\lambda-1)}}{A \lambda} \phi^{-\gamma(\lambda-1)}
$$

We noted that the index $n_{s}$ can be re-expressed in terms of the number $N$. Combining Eqs.(20) and (21) we obtain

$$
n_{s}=1-\frac{2}{A \lambda}\left[\frac{N}{A}+(A \lambda)^{\frac{-\lambda}{\lambda-1}}\right]^{-\frac{\lambda-1}{\lambda}} .
$$
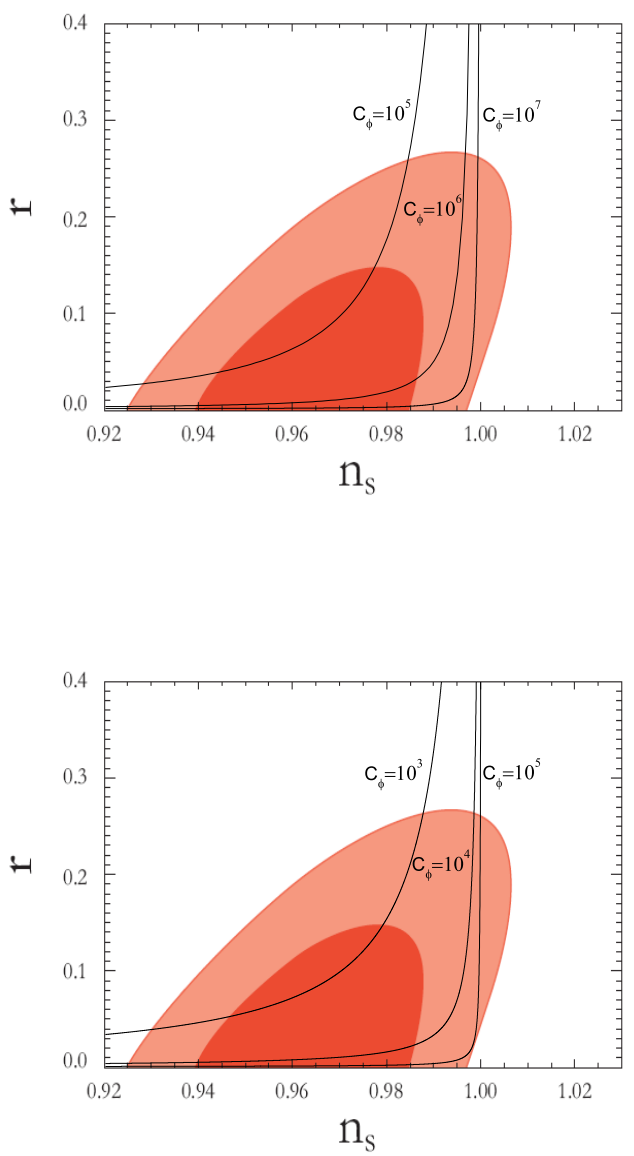

FIG. 1: Evolution of the tensor-scalar ratio $r$ versus the scalar spectrum index $n_{s}$ in the weak dissipative regime, for three different values of the parameter $C_{\phi}$. In the upper panel, we have take $\lambda=5$ and the lower panel, $\lambda=20$. In both panels we used $A=10^{-1}, \kappa=1$ and $C_{\gamma}=70$. 
As it was remarked in Ref.[30] the generation of tensor perturbations during inflation would produce gravitational wave. The corresponding spectrum is given by $\mathcal{P}_{g}=8 \kappa(H / 2 \pi)^{2}$. For this regime and from Eq.(22) we may write the tensor-scalar ratio $r$ as

$$
r(k)=\left(\frac{\mathcal{P}_{g}}{P_{\mathcal{R}}}\right) \simeq \beta_{5} \phi^{2},
$$

where $\beta_{5}=\frac{8 \kappa C_{\gamma}}{\pi^{2} C_{\phi}}$.

The tensor to scalar ratio can be written in terms of the scalar spectral index $n_{s}$ as

$$
r \simeq \beta_{5}\left[\frac{2 B^{-(\lambda-1)}}{A \lambda\left(1-n_{s}\right)}\right]^{\frac{2}{\gamma(\lambda-1)}} .
$$

Analogously, the tensor to scalar ratio as function of the number of e-folding $N$ results as

$$
r \simeq \beta_{5} B^{-2 / \gamma}\left[\frac{N}{A}+(A \lambda)^{\frac{-\lambda}{\lambda-1}}\right]^{\frac{2}{\lambda \gamma}} .
$$

In Fig.(1) we shows the dependence of the tensor-scalar ratio on the spectral index (for the weak regime) for the special case in which we fixe $A=10^{-1}$, and we have used three different values of the parameter $C_{\phi}$. In the upper panel we used $\lambda=5$ and in the lower panel $\lambda=20$.

The seven-year WMAP data places stronger limits on the tensor to scalar ratio $r$. In order to write down values that relate $n_{s}$ and $r$, we used Eq.(26). Also in both panels we have used the values $C_{\gamma}=70$ and $\kappa=1$. From the upper panel in which $\lambda=5$, we noted that for the value of the parameter $C_{\phi}>10^{5}$, the model is well supported by the data in the weak regime. Analogously, from de lower panel, we noted that for $C_{\phi}>10^{3}$, the model is well supported by the data. In general, we noted that when we increases the values of $\lambda$, the values of the parameters $C_{\phi}$ decreases (see Fig.(10). If we compared with respect to monomial potentials, the value of the parameter $C_{\phi}$, increase by two orders of magnitude and for the Hybrid models, $C_{\phi}$ increase by one order of magnitude, when $\lambda=5[31]$.

\section{THE STRONG DISSIPATIVE REGIME.}

We consider now the case in which $R=\Gamma / 3 H>1$. From Eqs.(7) and (13), we can obtained a relation between the scalar field and cosmological times given by

$$
\ln \left[\phi(t) / \phi_{0}\right]=\alpha_{1} \gamma_{\lambda}(t)
$$


where $\phi_{0}$ is a constant (without loss of generality we can taken $\phi_{0}=1$ ), $\alpha_{1}$ is defined by

$$
\alpha_{1}=\sqrt{\frac{2 \cdot 3^{1 / 4}}{\kappa \alpha_{0}}}(A \lambda)^{5 / 8}\left|(-4)^{1+\frac{5}{8}(\lambda-1)}\right|, \quad \text { and } \quad \gamma_{\lambda}(t) \equiv\left|\gamma\left[1+\frac{5}{8}(\lambda-1),-\frac{1}{4} \ln t\right]\right| .
$$

Here, $\gamma_{\lambda}(t)$ is the incomplete gamma function, see e.g. Ref.[32].

The Hubble parameter as a function of the inflaton field, $\phi$, is given by

$$
H(\phi)=A \lambda\left(\gamma_{\lambda}^{-1}\left[\frac{1}{\alpha_{1}} \ln \phi\right]\right)^{-1}\left(\ln \gamma_{\lambda}^{-1}\left[\frac{1}{\alpha_{1}} \ln \phi\right]\right)^{\lambda-1},
$$

where $\gamma_{\lambda}^{-1}\left[\frac{1}{\alpha_{1}} \ln \phi\right]$ is the inverse gamma function of $\gamma_{\lambda}(t)$.

At the last times, analogously to the case of the weak dissipative regime, the scalar potential from Eq.(12), becomes

$$
V(\phi)=\frac{K}{R^{2} \phi^{4}}\left(\gamma_{\lambda}^{-1}\left[\frac{1}{\alpha_{1}} \ln \phi\right]\right)^{-3}\left(\ln \gamma_{\lambda}^{-1}\left[\frac{1}{\alpha_{1}} \ln \phi\right]\right)^{3(\lambda-1) / 2},
$$

where $K=3^{1 / 2} \alpha_{0}^{2}(A \lambda)^{3 / 2} / \kappa$.

Considering Eq.(11) the dissipation coefficient, can be expressed as a function of the scalar field as

$$
\Gamma(\phi)=(3 A \lambda)^{3 / 4} \alpha_{0} \phi^{-2}\left(\gamma_{\lambda}^{-1}\left[\frac{1}{\alpha_{1}} \ln \phi\right]\right)^{-3 / 2}\left(\ln \gamma_{\lambda}^{-1}\left[\frac{1}{\alpha_{1}} \ln \phi\right]\right)^{\frac{3}{4}(\lambda-1)} .
$$

The dimensionless slow-roll parameter $\varepsilon$ for this regime, becomes

$$
\varepsilon=-\frac{\dot{H}}{H^{2}}=(A \lambda)^{-1}\left(\ln \gamma_{\lambda}^{-1}\left[\frac{1}{\alpha_{1}} \ln \phi\right]\right)^{-(\lambda-1)},
$$

and the slow-roll parameter $\eta$, is given by

$$
\eta=-\frac{\ddot{H}}{H \dot{H}}=(A \lambda)^{-1}\left(\ln \gamma_{\lambda}^{-1}\left[\frac{1}{\alpha_{1}} \ln \phi\right]\right)^{-\lambda}\left\{2\left(\ln \gamma_{\lambda}^{-1}\left[\frac{1}{\alpha_{1}} \ln \phi\right]\right)-(\lambda-1)\right\} .
$$

Again, following Ref.[13] the condition $\varepsilon=1$ at the beginning of inflation the scalar field, results

$$
\phi_{1}=\exp \left[\alpha_{1} \gamma_{\lambda}\left(e^{\mu}\right)\right]
$$

where $\mu \equiv(A \lambda)^{\frac{-1}{\lambda-1}}$.

The number of e-folds $N$ in this regime, from Eq.(28) is given by

$$
N=\int_{t_{1}}^{t_{2}} H d t=A\left\{\left(\ln \gamma_{\lambda}^{-1}\left[\frac{1}{\alpha_{1}} \ln \phi_{2}\right]\right)^{\lambda}-\left(\ln \gamma_{\lambda}^{-1}\left[\frac{1}{\alpha_{1}} \ln \phi_{1}\right]\right)^{\lambda}\right\} .
$$


In the case of high dissipation regime and following Ref.[33], we can write $\delta \phi^{2} \simeq \frac{k_{F} T}{2 \pi^{2}}$, where the wave-number $k_{F}=\sqrt{\Gamma H}=H \sqrt{3 R}>H$, and corresponds to the freeze-out scale at which dissipation damps out to the thermally excited fluctuations. From Eqs.(28) and (31) we obtained that

$$
\mathcal{P}_{\mathcal{R}}=\alpha_{2} \phi^{-3}\left(\gamma_{\lambda}^{-1}\left[\frac{1}{\alpha_{1}} \ln \phi\right]\right)^{-9 / 4}\left(\ln \gamma_{\lambda}^{-1}\left[\frac{1}{\alpha_{1}} \ln \phi\right]\right)^{\frac{15}{8}(\lambda-1)}
$$

where

$$
\alpha_{2}=3^{3 / 8} \alpha_{0}^{3 / 2}\left(4 \pi^{2}\right)^{-1}\left(\frac{\kappa^{3}}{2 C_{\gamma}}\right)^{1 / 4}(A \lambda)^{15 / 8} .
$$

Considering Eqs.(28) and (31) the scalar spectral index $n_{s}=d \mathcal{P}_{\mathcal{R}} / d \ln k$, is given by

$$
n_{s}=1-\frac{9}{4 A \lambda}\left(\ln \gamma_{\lambda}^{-1}\left[\frac{1}{\alpha_{1}} \ln \phi\right]\right)^{-(\lambda-1)} .
$$

Analogously as the weak regime, the scalar spectra index, can be write in terms of the number of e-folds. Considering Eqs. (34) and (35) results

$$
n_{s}=1-\frac{9}{4 A \lambda}\left[\frac{N}{A}+(A \lambda)^{\frac{-\lambda}{\lambda-1}}\right]^{-\frac{\lambda-1}{\lambda}}
$$

For this regime we may write the tensor-scalar ratio, using Eqs. (29) and (36), we get

$$
r(k)=\left(\frac{\mathcal{P}_{g}}{P_{\mathcal{R}}}\right)=\alpha_{3} \phi^{3}\left(\gamma_{\lambda}^{-1}\left[\frac{1}{\alpha_{1}} \ln \phi\right]\right)^{1 / 4}\left(\ln \gamma_{\lambda}^{-1}\left[\frac{1}{\alpha_{1}} \ln \phi\right]\right)^{\frac{\lambda-1}{8}}
$$

where $\alpha_{3}=\frac{2 \kappa(A \lambda)^{2}}{\pi^{2} \alpha_{2}}$.

Analogously as the weak regimen, the tensor to scalar ratio $r$, in terms of the scalar spectral index, becomes

$$
r=\alpha_{3}\left(F\left[n_{s}\right]\right)^{\frac{\lambda-1}{8}} \exp \left[\frac{F\left[n_{s}\right]}{4}+3 \alpha_{1} \gamma_{\lambda}\left(e^{F\left[n_{s}\right]}\right)\right]
$$

where

$$
F\left[n_{s}\right] \equiv\left[\frac{9}{4 A \lambda\left(1-n_{s}\right)}\right]^{\frac{1}{\lambda-1}} .
$$

Also, we can write the tensor-scalar ratio as function of the number of e-folding

$$
r=\alpha_{3}(G[N])^{\frac{\lambda-1}{8}} \exp \left[\frac{G[N]}{4}+3 \alpha_{1} \gamma_{\lambda}\left(e^{G[N]}\right)\right]
$$

where $G[N] \equiv\left[\frac{N}{A}+(A \lambda)^{\frac{-\lambda}{\lambda-1}}\right]^{1 / \lambda}$. 

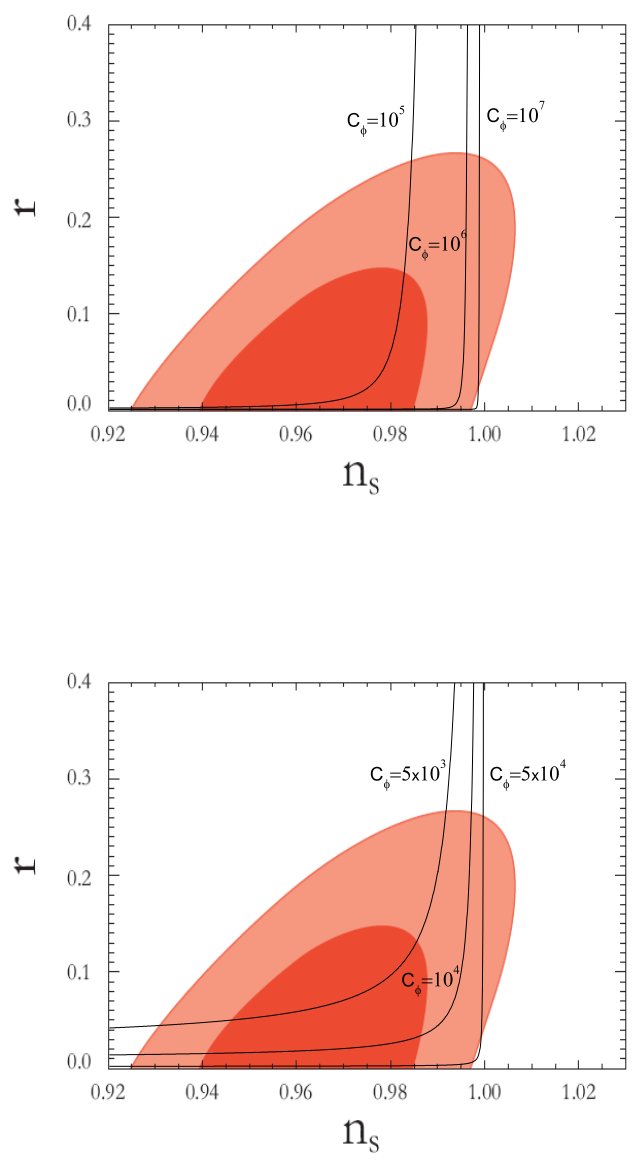

FIG. 2: Evolution of the tensor-scalar ratio $r$ versus the scalar spectrum index $n_{s}$ in the strong dissipative regime, for three different values of the parameter $C_{\phi}$. In the upper panel we have used $\lambda=5$ and the lower panel $\lambda=20$. In both panels we used $A=10^{-1}, \kappa=1$ and $C_{\gamma}=70$.

For the strong dissipative regime i.e., $R=\Gamma / 3 H>1$, the Fig.(2) shows the dependence of the tensor-scalar ratio on the spectral index for the special case in which we fixe $A=10^{-1}$, and we have used three different values of the parameter $C_{\phi}$. In the upper panel we used $\lambda=5$ and in the lower panel $\lambda=20$.

In order to write down values that relate $n_{s}$ and $r$, we used Eq.(40). Also in both panels we have used the values $C_{\gamma}=70$ and $\kappa=1$. From the upper panel in which $\lambda=5$, we noted that for the value of the parameter $C_{\phi}>10^{4}$, the model is well supported by the data in the weak regime. Analogously, from de lower panel in which $\lambda=20$, we noted that for $C_{\phi}>10^{2}$, the model is well supported by the data. Analogous as the weak regime, we noted 
that when we increases the values of $\lambda$, the values of the parameter $C_{\phi}$ decreases. Analogous as the weak scenario, if we compared with respect to monomial potentials, the value of the parameter $C_{\phi}$ increase by three orders of magnitude and for the Hybrid models, $C_{\phi}$ increase by two orders of magnitude [31], when $\lambda=5$.

\section{CONCLUSIONS}

In this paper we have studied the warm-logamediate inflationary model in the weak and strong dissipative regimes. In the slow-roll approximation we have found explicit expressions for the corresponding dissipation parameter $\Gamma$, scalar potential $V$, the number of e-folds $N$, power spectrum of the curvature perturbations $P_{\mathcal{R}}$, tensor-scalar ratio $r$ and scalar spectrum index $n_{s}$.

When $\Gamma<3 H$ warm inflation occurs in the so-called weak dissipative regime. In this case, the dissipation coefficient $\Gamma \propto \phi^{-8} \exp \left[-3 B \phi^{\gamma}\right]$ and the scalar potentia $V(\phi) \propto$ $\phi^{\alpha} \exp \left[-\beta \phi^{\gamma}\right]$ that coincides with Ref.[13]. In order to bring some explicit results we have taken trajectories for different combinations of the parameters in the $r-n_{s}$ plane to firstorder in the slow roll approximation. We noted that the parameter $C_{\phi}$, which is bounded from bellow, $C_{\phi}>10^{5}$, the model is well supported by the data as could be seen from upper panel of Fig.(1) in which $\lambda=5$. We also noted that for the lower panel where $\lambda=20$, the values of the parameter $C_{\phi}>10^{3}$ the model is well supported by the data. In both panel, we have used the WMAP seven year data and also we have taken the value $A=10^{-1}, \kappa=1$ and $C_{\gamma}=70$. On the other hand, when $\Gamma>3 H$ warm inflation occurs in the so-called strong dissipative regime. In this regime, the effective potential is given by Eq.(30) and the dissipation coefficient $\Gamma$ by Eq.(31). Analogously as the weak regime the Fig.(2) shows trajectories for different combinations of the parameters $C_{\phi}$ in the $r-n_{s}$ plane, for $A=10^{-1}$ and $C_{\gamma}=70$. Curiously, in both regimes we noted that when we increases the values of $\lambda$, the values of the parameter $C_{\phi}$ decreases.

In this paper, we have not addressed the non-Gaussian effects from the non-linearity parameter $f_{N L}$, during warm-logamediate inflation (see e.g., Refs. [26, 34]). We hope to return to this point in the near future. 


\section{Acknowledgments}

R.H. was supported by COMISION NACIONAL DE CIENCIAS Y TECNOLOGIA through FONDECYT grants $\mathrm{N}^{0} 1090613$ and $\mathrm{N}^{0}$ 1110230. M.O. was supported by Proyecto D.I. PostDoctorado 2012 PUCV.

[1] A. Guth , Phys. Rev. D 23, 347 (1981); A.A. Starobinsky, Phys. Lett. B 91, 99 (1980); A.D. Linde, Phys. Lett. B 108, 389 (1982); idem Phys. Lett. B 129, 177 (1983); A. Albrecht and P. J. Steinhardt, Phys. Rev. Lett. 48,1220 (1982); K. Sato, Mon. Not. Roy. Astron. Soc. 195, 467 (1981).

[2] V.F. Mukhanov and G.V. Chibisov, JETP Letters 33, 532(1981); S. W. Hawking, Phys. Lett. B 115, 295 (1982); A. Guth and S.-Y. Pi, Phys. Rev. Lett. 49, 1110 (1982); A. A. Starobinsky, Phys. Lett. B 117, 175 (1982); J.M. Bardeen, P.J. Steinhardt and M.S. Turner, Phys. Rev.D 28, 679 (1983).

[3] D. Larson et al., Astrophys. J. Suppl. 192, 16 (2011).

[4] C. L. Bennett et al., Astrophys. J. Suppl. 192, 17 (2011); N. Jarosik et al., Astrophys. J. Suppl. 192, 14 (2011)

[5] A. Berera, Phys. Rev. Lett. 75, 3218 (1995); A. Berera, Phys. Rev. D 55, 3346 (1997).

[6] L.M.H. Hall, I.G. Moss and A. Berera, Phys.Rev.D 69, 083525 (2004); I.G. Moss, Phys.Lett.B 154, 120 (1985); A. Berera and L.Z. Fang, Phys.Rev.Lett. 741912 (1995); A. Berera, Nucl.Phys B 585, 666 (2000).

[7] A. Berera, Phys. Rev.D 54, 2519 (1996).

[8] A. Berera, Phys. Rev. D 55, 3346 (1997); J. Mimoso, A. Nunes and D. Pavon, Phys.Rev.D 73, 023502 (2006); R. Herrera, S. del Campo and C. Campuzano, JCAP 10, 009 (2006); S. del Campo, R. Herrera and D. Pavon, Phys. Rev. D 75, 083518 (2007); S. del Campo and R. Herrera, Phys. Lett. B 653, 122 (2007); M. A. Cid, S. del Campo and R. Herrera, JCAP 10, 005 (2006); J. C. B. Sanchez, M. Bastero-Gil, A. Berera and K. Dimopoulos, Phys. Rev. D 77123527 (2008); R. Herrera, Phys. Rev. D81, 123511 (2010); R. Herrera, E. San Martin, Eur. Phys. J. C71, 1701 (2011).

[9] R. Brandenberger and M. Yamaguchi, Phys. Rev. D 68, 023505 (2003). 
[10] M. Bastero-Gil, A. Berera, R. O. Ramos and J. G. Rosa, arXiv:1110.3971 [hep-ph].

[11] J. D. Barrow, Class. Quant. Grav. 13, 2965 (1996).

[12] J. Yokoyama and K. Maeda, Phys. Lett. B 207, 31 (1988).

[13] J. D. Barrow and N. J. Nunes, Phys. Rev. D 76043501 (2007).

[14] D. H. Lyth and A. Riotto, Phys. Rept. 314, 1 (1999).

[15] A. D. Linde (2005), hep-th/0503203.

[16] P.G. Ferreira, M. Joyce, Phys.Rev.D 58, 023503 (1998).

[17] P. Binetruy, Phys.Rev.D 60, 063502 (1999).

[18] P. Parsons and J. D. Barrow, Phys. Rev. D 51, 6757 (1995). 2005) 1.

[19] Ph. Brax, J. Martin, Phys.Lett.B 468, 40 (1999).

[20] S. del Campo, R. Herrera, J. Saavedra, C. Campuzano, E. Rojas, Phys. Rev. D80, 123531 (2009).

[21] P.J.E. Peebles, B. Ratra, Rev.Mod.Phys.75, 559 (2003); R. Ghosh, S. Chattopadhyay, U. Debnath, arXiv:1105.4538 [gr-qc]]; U. Debnath, S. Chattopadhyay, M. Jamil, arXiv:1107.0541 [physics.gen-ph]].

[22] G. Calcagni and G. Nardelli, Int. J. Mod. Phys. D 19, 329 (2010).

[23] G. Calcagni and G. Nardelli, Nucl. Phys. B 823, 234 (2009)

[24] I. G. Moss and C. Xiong, arXiv:hep-ph/0603266.

[25] A. Berera, M. Gleiser and R. O. Ramos, Phys. Rev. D 58123508 (1998); A. Berera and R. O. Ramos, Phys. Rev. D 63, 103509 (2001).

[26] J. C. Bueno Sanchez, M. Bastero-Gil, A. Berera and K. Dimopoulos, Phys. Rev. D 77, 123527 (2008).

[27] A. Berera, I. G. Moss and R. O. Ramos, Rept. Prog. Phys. 72, 026901 (2009).

[28] M. Bastero-Gil, A. Berera and R. O. Ramos, JCAP 1109, 033 (2011).

[29] A. Berera, Nucl. Phys. B 585, 666 (2000).

[30] K. Bhattacharya, S. Mohanty and A. Nautiyal, Phys.Rev.Lett. 97, 251301 (2006).

[31] M. Bastero-Gil and A. Berera, Int. J. Mod. Phys. A 24, 2207 (2009)

[32] Abramowitz, M. and Stegun, I. A. (Eds.). Handbook of Mathematical Functions with Formulas, Graphs, and Mathematical Tables, 9th printing. New York: Dover, 1972; Arfken, G. "The Incomplete Gamma Function and Related Functions." Mathematical Methods for Physicists, 
3rd ed. Orlando, FL: Academic Press, 1985.

[33] A. Taylor and A. Berera, Phys. Rev. D 69, 083517 (2000).

[34] S. Gupta, A. Berera, A. F. Heavens and S. Matarrese, Phys. Rev. D 66, 043510 (2002); I. G. Moss and C. Xiong, JCAP 0704, 007 (2007). 